\title{
Posterior interosseous nerve compression secondary to a parosteal lipoma: Case report and literature review
}

\author{
Ronen Avram BSc MD, Nicolas M Hynes MSc MD FRCSC
}

\begin{abstract}
R Avram, NM Hynes. Posterior interosseous nerve compression secondary to a parosteal lipoma: Case report and literature review. Can J Plast Surg 2004;12(2):69-72.

An unusual case of limited radial nerve palsy secondary to a parosteal lipoma is presented, along with a thorough review of the literature. Palsy of the posterior interosseous nerve secondary to compression by a lipoma is a rare occurrence. Most cases tend to occur in the fifth to eighth decades of life. The pattern of physical symptoms may be quite variable. A high degree of suspicion must be maintained in the setting of unexplained symptoms. The literature review revealed the presence of a diagnostic proximal forearm radiolucent mass in 13 of 15 patients. Although they are used as diagnostic aids, computed tomography and magnetic resonance imaging have not been shown to alter management. Though benign tumours constitute a minority of such cases, a plain radiograph is recommended in addition to electromyographic and nerve conduction studies.
\end{abstract}

Key Words: Parosteal lipoma; Posterior interosseous nerve palsy; Radial nerve palsy

\section{Compression du nerf interosseux postérieur, secondaire à un lipome parostéal : exposé de cas et examen de la documentation}

\begin{abstract}
Voici la description d'un cas plutôt rare de paralysie partielle du nerf radial, secondaire à un lipome parostéal, suivie d'un examen exhaustif de la documentation. La compression du nerf interosseux postérieur par un lipome donne rarement lieu à la paralysie. La plupart des cas se rencontrent chez les personnes âgées entre 50 et 80 ans. Les symptômes physiques peuvent varier considérablement. Il faut entretenir des doutes importants devant des symptômes inexpliqués. L'examen de la documentation a révélé la présence diagnostique d'une masse radiotransparente dans l'avant-bras proximal chez 13 patients sur 15. Bien qu'elles servent d'outils d'aide au diagnostic, la tomodensitométrie et l'imagerie par résonance magnétique ne modifient pas pour autant la prise en charge. Même si les tumeurs bénignes sont rares en pareils cas, il est recommandé de procéder à une radiographie simple, outre l'électromyographie et l'exploration de la conduction nerveuse.
\end{abstract}

Spontaneous palsy of the posterior interosseous nerve (PIN) $\checkmark$ is an uncommon clinical condition. Tumours comprise only a small fraction of the potential etiologies. Atraumatic palsy of the PIN is by and large a result of compression from adjacent anatomical structures such as the arcade of Frohse, the extensor carpi radialis brevis and the vascular leash of Henry. Palsy of the PIN secondary to compression by a lipoma is a rare occurrence. A review of the English literature reveals fewer than 30 such cases.

Compression of the PIN secondary to a lipoma was first described by Richmond in 1953 (1). His report involved a patient with an inability to extend the index finger and thumb. The largest series published thus far was put forth by Barber et al in 1962 (2). They reviewed the clinical records of 32 patients in whom the diagnosis of a benign soft tissue tumour with neurological dysfunction of an extremity was made. Eight of these patients were found to have a lipoma causing compression of the radial nerve, though two of these cases were previously reported by Hustead et al in 1958 (3) and another two resulted in sensory deficits alone via compression of the radial nerve against the humerus. There are several additional reports $(2,4-14)$. Jebson et al (8) described an unusual intraosseous lipoma with extraosseous extension and a secondary PIN palsy. The most recent report of a PIN palsy secondary to a lipoma was produced by Fitzgerald et al in December 2002 (7).

The current paper presents the case of a 69-year-old man with a parosteal lipoma of the left forearm resulting in compression of PIN branches to the extensor digitorum communis (EDC) muscle. His condition was partially masked by apparent subluxation of his extensor tendons at the third and fourth metacarpophalangeal (MCP) joints, a common sequela of his underlying arthritic condition.

\section{CASE PRESENTATION}

A 69-year-old right-hand-dominant man presented with a four-month history of progressive difficulty with extending his left long and ring fingers. He was a poor historian but did not recall any specific history of trauma. His past medical history was significant for psoriatic arthritis. On initial examination, the patient demonstrated an extensor lag of 85 degrees for the left long finger and 80 degrees for the left ring finger at the level of the MCP joint (Figure 1). His extensor tendons were found to be subluxing at the MCP level and he was brought to the operating room for reconstruction of his extensor

Division of Plastic Surgery, Department of Surgery, St Joseph's Healthcare and McMaster University, Hamilton, Ontario

Correspondence: Dr Nicolas M Hynes, 183 Hughson Street South, second floor, Hamilton, Ontario L8N 2B6. Telephone 905-645-5640,

fax905-645-5643, e-mail hynesplasticsurgery@sympatico.ca 


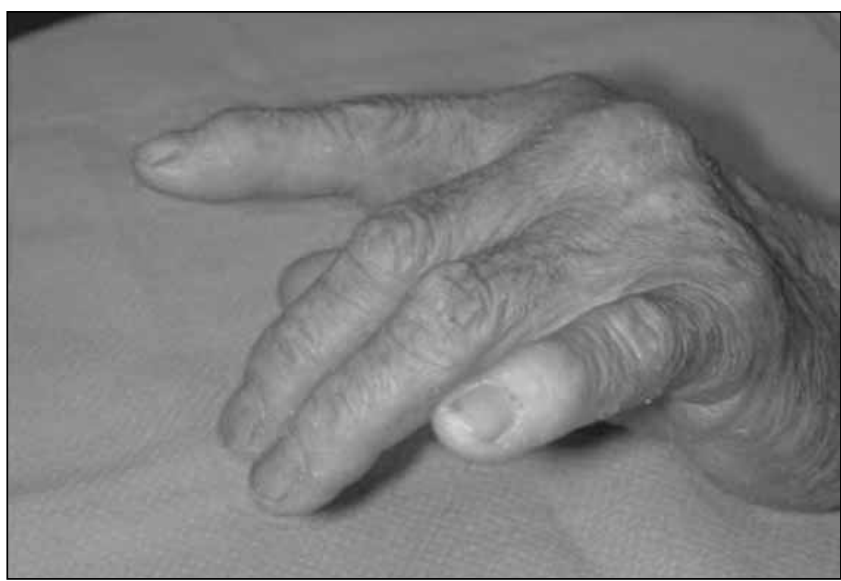

Figure 1) Extensor lag of the long and ring finger metacarpophalangeal joints

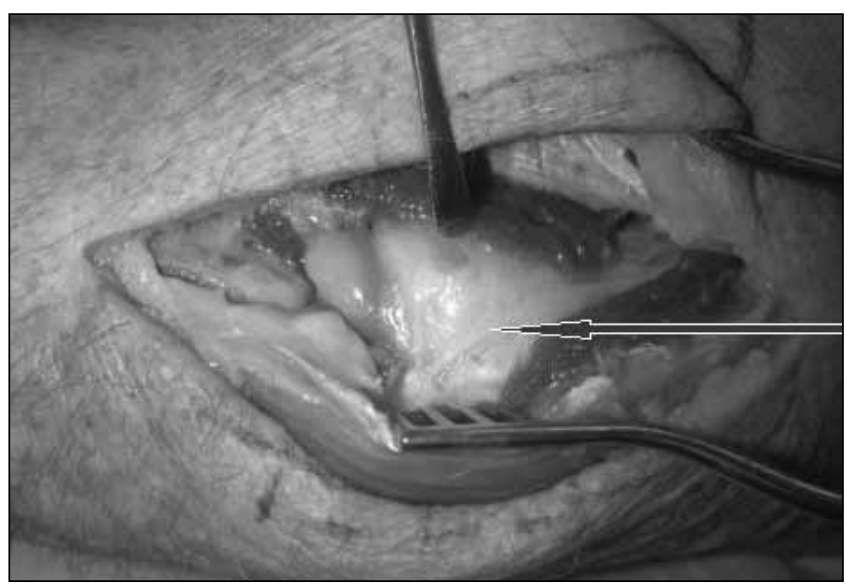

Figure 2) Lipoma underneath the supinator muscle impinging on the posterior interosseous nerve branch (arrow) to the extensor digitorum communis muscle

apparatus. Immediately preceding his scheduled operation, re-examination could not confirm the previously detected subluxation, although the deficit persisted. The patient's left hand was explored nonetheless with the belief that his extensor tendons may have ruptured. Operative findings included a moderately inflamed extensor synovium at the level of the dorsal retinaculum but no tendon rupture. A tenolysis was performed and a biopsy of the tenosynovium was sent for pathology. This revealed fibrosis and nonspecific mild chronic inflammation.

Despite rigorous postoperative physiotherapy, the patient's condition failed to improve. Re-examination at three months following surgery revealed tenderness in the proximal forearm overlying the region of the radial tunnel. The patient was returned to the operating room for exploration of his radial nerve. Operative findings included atrophy of the EDC muscle fibres and a large space-occupying lesion beneath the supinator muscle interposed between the radius and several fascicles of the PIN (Figures 2, 3). The lesion was consistent with a lipoma and was $5 \mathrm{~cm}$ in length. It was carefully excised under loupe magnification. Pathology examination confirmed the intraoperative diagnosis of lipoma.

\section{Postoperative evaluation}

There was no change in function six weeks postoperatively and only slight improvement was noted at the 3.5-month

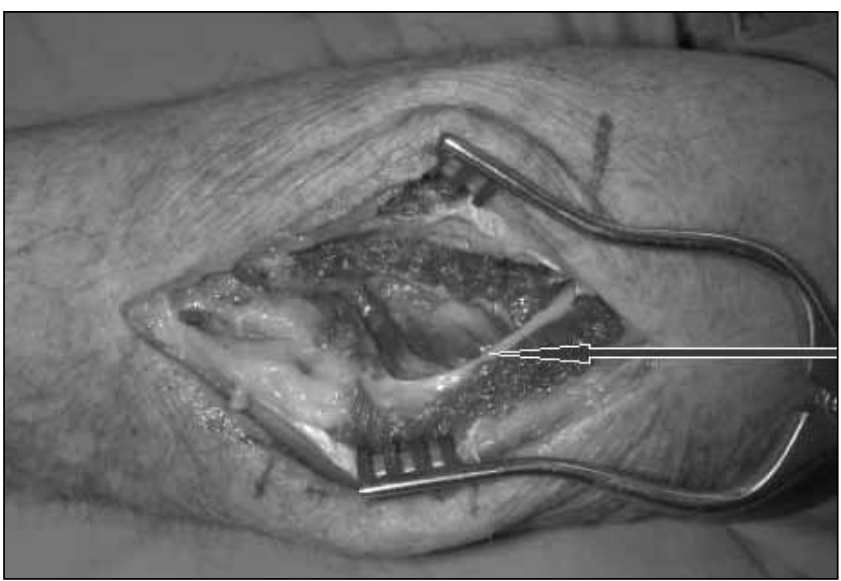

Figure 3) Postresection of lipoma with preservation of the motor nerve branch (arrow) to the extensor digitorum communis muscle

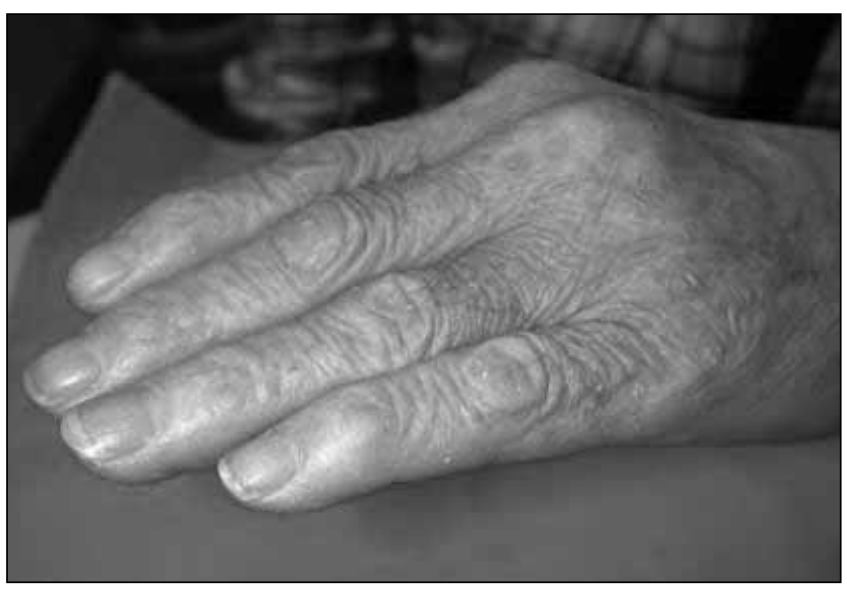

Figure 4) Postoperative result

post-operative visit. However, full extension of the ring finger was achieved by the eighth postoperative month, with only a 20 degree extension lag of the long finger (Figure 4).

\section{DISCUSSION}

Palsy of the PIN in the absence of trauma is an uncommon clinical entity. Compression of the PIN secondary to a lipoma comprises a minute fraction of the latter. Deep-seated lipomas are rare neoplasms. They have been classified as intermuscular, intramuscular, parosteal and intraosseous. In the largest independent series, conducted by Barber et al in 1962 (2), all eight lipomas described exerted their effect by compressing the nerve against the underlying humerus or radius, implying an intra- or intermuscular origin of the lipoma. Our case was a parosteal lipoma. Review of the available details in the English literature reveals only 18 such reported cases (Table 1). Further isolating our case from the previously reported cases is the exclusive compression of the PIN branches to the EDC muscle. All descriptions reported to date suggest that multiple PIN innervated muscle groups are likely to be affected. None have described weakness of the long and ring fingers alone.

Diagnosis of such a lesion can prove to be quite challenging. A high level of suspicion must be maintained, particularly 
TABLE 1

Literature review

\begin{tabular}{|c|c|c|c|c|c|c|c|c|}
\hline Case & e Year & $\begin{array}{c}\text { Author } \\
\text { (reference) }\end{array}$ & Age & Sex & Symptoms & $\begin{array}{l}\text { Location } \\
\text { of tumour }\end{array}$ & $\begin{array}{c}\text { Duration of } \\
\text { motor symptoms }\end{array}$ & Roentgenogram \\
\hline 1 & 1953 & Richmond (1) & 62 & M & Left thumb and index & Parosteal & 3 months & Radiolucency in proximal forearm \\
\hline 2 & 1954 & Campbell and Wulf (6) & 40 & $\mathrm{~F}$ & Left thumb and index & Parosteal & 3 months & Not performed \\
\hline 3 & 1955 & Hustead et al (3) & 63 & $\mathrm{~F}$ & Left thumb, then all digits and wrist & Parosteal & 1.5 years & Radiolucency in proximal forearm \\
\hline 4 & 1955 & Hustead et al (3) & 56 & M & $\begin{array}{l}\text { Right long finger, then all digits } \\
\text { and wrist }\end{array}$ & Parosteal & 1.5 years & Not performed \\
\hline 5 & 1962 & White and Hanna (22) & 65 & $\mathrm{~F}$ & Right little and ring fingers & Parosteal & 5 years & Not performed \\
\hline 6 & 1962 & Barber et al (2) & NA & NA & NA & NA & NA & NA \\
\hline 7 & 1962 & Barber et al (2) & NA & NA & NA & NA & NA & NA \\
\hline 8 & 1962 & Barber et al (2) & NA & NA & NA & NA & NA & NA \\
\hline 9 & 1964 & Moon and Marmor (19) & 73 & M & Left thumb, then all digits and wrist & Parosteal & 8 years & Radiolucency in proximal forearm \\
\hline 10 & 1966 & Capener (15) & 58 & M & All digits & NA & 2 months & Not performed \\
\hline 11 & 1966 & Sharrard (13) & 30 & M & All digits and wrist & Parosteal & 1 year & Calcified tumour in forearm \\
\hline 12 & 1971 & Phalen et al (23) & 56 & M & All digits & NA & 5 years & Not diagnostic \\
\hline 13 & 1971 & Phalen et al (23) & 62 & M & Right little, ring and middle fingers & NA & 1 year & Radiolucency in proximal forearm \\
\hline 14 & 1972 & Leffert (9) & NA & NA & NA & NA & NA & NA \\
\hline 15 & 1973 & Berry and Moiel (4) & 30 & M & $\begin{array}{l}\text { All digits affected and extensor } \\
\text { carpi ulnaris }\end{array}$ & Parosteal & 18 months & Not diagnostic \\
\hline 16 & 1974 & Wu et al (14) & 77 & M & All fingers and wrist extensors & Parosteal & 12 years & Radiolucency in proximal forearm \\
\hline 17 & 1985 & Pidgeon et al (18) & 45 & M & All fingers, ulnar wrist extensor & Intermuscular & 3 months & Radiolucency in proximal forearm \\
\hline 18 & 1986 & Bieber et al (5) & 53 & $\mathrm{~F}$ & All digits & Parosteal & NA & Radiolucency in antecubital fossa \\
\hline 19 & 1992 & Lidor et al (10) & 40 & $\mathrm{~F}$ & Complete extensor paralysis & Parosteal & 4 months & Radiolucency in proximal forearm \\
\hline 20 & 1992 & Lidor et al (10) & 72 & $\mathrm{~F}$ & Complete extensor paralysis & Parosteal & 2 years & Radiolucency in proximal forearm \\
\hline 21 & 1996 & Hashizume et al (16) & NA & NA & NA & NA & NA & NA \\
\hline 22 & 1998 & Nishida et al (12) & 60 & $\mathrm{~F}$ & All digits and wrist & Parosteal & 2 months & Not performed \\
\hline 23 & 1998 & Nishida et al (12) & 61 & $\mathrm{~F}$ & All fingers and wrist & Parosteal & NA & Radiolucency in proximal forearm \\
\hline 24 & 2002 & Jebson et al (8) & 70 & M & Left thumb and fingers & Intraosseous & 4 months & Radiolucency in proximal forearm \\
\hline 25 & 2002 & Fitzgerald et al (7) & 71 & $\mathrm{~F}$ & Thumb and index & Parosteal & $<1$ month & Radiolucency in proximal forearm \\
\hline 26 & 2002 & Fitzgerald et al (7) & 64 & M & Wrist, long, ring and small digits & Parosteal & 3 months & Radiolucency in proximal forearm \\
\hline 27 & 2002 & Fitzgerald et al (7) & 68 & $\mathrm{~F}$ & Thumb and index & Parosteal & 5 months & Radiolucency in proximal forearm \\
\hline 28 & 2002 & Fitzgerald et al (7) & 63 & $\mathrm{~F}$ & All digits & Parosteal & 2 years & Radiolucency in proximal forearm \\
\hline 29 & 2002 & $\begin{array}{l}\text { Avram and Hynes } \\
\text { (current case) }\end{array}$ & 69 & M & Left middle and ring fingers & Parosteal & 4 months & Not performed \\
\hline
\end{tabular}

F Female; M Male; NA Not available

when uncertainty about the diagnosis exists. Despite assertions by multiple authors regarding the sex predilection of the disease towards female patients $(4,7)$, our search revealed a relatively equal distribution between males and females. Most cases tend to occur in the fifth to eighth decades of life. The pattern of physical symptoms may be quite erratic. The presence of pain is variable $(1,5,10,16)$. Extensor function is variably affected as well. Often there is no palpable mass or tenderness.

Though advocated by many, nerve conduction studies and electromyography are not routinely performed. Four of the eight most recently published studies did not employ either test $(5,7,8,10,12,16-18)$. When performed, the results serve to confirm a disturbance of the PIN and its associated muscle groups. The presence of a space-occupying lesion, however, cannot be confirmed by these measures. Isolated nerve branch involvement may remain undetected as well.

What appears fairly consistently throughout the literature, however, is the presence of an associated radiolucent mass on radiography. When performed, a diagnostic, proximal forearm, radiolucent mass was discovered in 13 of 15 patients $(5,7,8,10,11,13,18-20)$. In our case, a plain radiograph was not performed and certainly this could have been of diagnostic benefit.

More recently published studies have employed additional imaging modalities such as ultrasonography, computed tomography $(\mathrm{CT})$ and magnetic resonance imaging (MRI). In all instances, imaging was preceded by a palpable mass on physical examination. Though significantly cheaper and readily available, ultrasonography is superceded by CT and MRI in terms of visual discrimination. CT can be very useful for delineating tumour boundaries but MRI is the preferred modality for evaluation of soft tissue neoplasms. The signal intensity of a lipoma is identical to that of subcutaneous fat on T1-weighted images. MRI may also distinguish muscle atrophy associated with a compression neuropathy (20). The clinical usefulness of such diagnostic modalities must be weighed against valued health care resources. None of the studies have demonstrated a shift in management based on CT or MRI. Absence of a palpable mass in the setting of a PIN palsy should prompt an $\mathrm{x}$-ray image at most.

Finally, persistence of symptoms in the absence of a diagnosis should prompt early surgical exploration of the PIN. Failure 
to do so may result in permanent dysfunction of hand and/or wrist extensors. When symptoms persisted beyond five years, decompression of the PIN did not result in significant recovery of the affected extensors $(14,19)$. In the case reported by Jebson et al (8), tendon transfers were carried out at the time of tumour excision. A more recent review conducted by Thoma et al (21) recommends immediate tendon transfers when recovery of the PIN is considered doubtful despite decompression. In their experience, a severe hourglass appearance of the PIN at the site of compression was an indication to perform transfers.

\section{CONCLUSION}

Among previously cited etiologies of spontaneous PIN palsy, compression secondary to a benign tumour remains relatively uncommon. Compression of the radial nerve by surrounding anatomic structures, particularly the arcade of Frohse, remains the most common cause of atraumatic PIN palsy. Surgical

\section{REFERENCES}

1. Richmond DA. Lipoma causing a posterior interosseous nerve lesion. J Bone Joint Surg Br 1953;35B:83.

2. Barber KW, Bianco AJ, Soule EH, MacCarty CS. Benign extraneural soft-tissue tumors of the extremities causing compression of nerves. J Bone Joint Surg Am 1962;44A:98-104.

3. Hustead AP, Mulder DW, MacCarty CS. Nontraumatic, progressive paralysis of the deep radial (posterior interosseous) nerve. AMA Arch Neurol Psychiatry 1958;79:269-74.

4. Berry JB, Moiel RH. Parosteal lipoma producing paralysis of the deep radial nerve. Southern Med J 1973;66:1298-300.

5. Bieber EJ, Moore JR, Weiland AJ. Lipomas compressing the radial nerve at the elbow. J Hand Surg (Am) 1986;11A:533-5.

6. Campbell CS, Wulf RF. Lipoma producing a lesion of the deep branch of the radial nerve. J Neurosurg 1954;11:310-1.

7. Fitzgerald A, Anderson W, Hooper G, Posterior interosseous nerve palsy due to parosteal lipoma. J Hand Surg (Br) 2002;27:535-7.

8. Jebson PJL, Schock EJ, Biermann JS. Intraosseous lipoma of the proximal radius with extraosseous extension and a secondary posterior interosseous nerve palsy. Am J Orthopedics 2002;31:413-6.

9. Leffert RD. Lipomas of the upper extremity. J Bone Joint Surg Am 1972;54A:1262-6.

10. Lidor C, Lotem M, Hallel T. Parosteal lipoma of the proximal radius: A report of five cases. J Hand Surg (Am) 1992;17:1095-7.

11. Mulholland RC. Non-traumatic progressive paralysis of the posterior interosseous nerve. J Bone Joint Surg Br 1966;48:781-5.

12. Nishida J, Shimamura T, Ehara S, et al. Posterior interosseous nerve exploration is often recommended as both a diagnostic and therapeutic maneuvre.

We presented the rare case of PIN palsy secondary to compression by a parosteal lipoma. EDC muscle fibres were independently affected. The clinical picture was partially masked by intact and unaffected extensor digiti minimi and extensor indicis proprius musculature as well as apparent extensor subluxation at the third and fourth MCP joints. Review of the literature reveals fewer than 30 cases over the past 50 years. There is a variable pattern of presentation with more than one extensor muscle group usually affected. Radiography has been shown to be helpful in diagnosis. A delay in treatment may result in permanent dysfunction, though near-complete to complete recovery may be expected in patients with symptoms having lasted up to five years.

Therefore, early surgical exploration is indicated in the setting of spontaneous, unexplained PIN palsy. Plain radiographs are recommended in addition to electromyography and nerve conduction studies.

palsy caused by parosteal lipoma of proximal radius. Skeletal Radiol 1998;27:375-9.

13. Sharrard WJW. Posterior interosseous neuritis. J Bone Joint Surg 1966;48B:777-80.

14. Wu KT, Jordon FR, Eckert C. Lipoma, a cause of paralysis of deep radial (posterior interosseous) nerve: Report of a case and review of the literature. Surgery 1974;75:790-5.

15. Capener N. The vulnerability of the posterior interosseous nerve of the forearm. J Bone Joint Surg Br 1966;48:770-3.

16. Hashizume H, Nishida K, Nanba Y, Shigeyama Y, Inoue H, Morito Y. Non-traumatic paralysis of the posterior interosseous nerve. J Bone Joint Surg Br 1996;78:771-6.

17. Carfi J, Dong M. Posterior interosseous syndrome revisited. Muscle Nerve 1985;8:499-502.

18. Pidgeon KJ, Abadee P, Kanakamedala R, Uchizono M. Posterior interosseous nerve syndrome caused by an intermuscular lipoma. Arch Phys Med Rehabil 1985;66:468-71.

19. Moon N, Marmor L. Parosteal lipoma of the proximal part of the radius. J Bone Joint Surg Am 1964;46:608-14.

20. Yu JS, Weis L, Becker W. MR imaging of a parosteal lipoma. J Clin Imaging 2000;24:15-8.

21. Thoma A, Ching S, Nelluri P. Outcomes of surgical treatment in posterior interosseous nerve syndrome Can J Plast Surg 2002;10:210-3.

22. White WL, Hanna DC. Troublesome lipomata of the upper extremity. J Bone Joint Surg 1962;44A:4:1353-9.

23. Phalen GS, Kendrick JI, Rodriguez JM. Lipomas of the upper extremity. A series of fifteen tumors in the hand and wrist and six tumors causing nerve compression. Am J Surg 1971;121:298-306. 